\title{
A COMPARISON OF SOLAR AND METEORITIC ABUNDANCES
}

\author{
H. HOLWEGER
}

Recent solar abundance data confirm the relationship between the sun and carbonaceous chondrites. New results for the solar $5 / \mathrm{Ca}$ and $\mathrm{Na} / \mathrm{Ca}$ ratio discriminate between types 1, 2, and 3. These and interstellar properties pose constraints on the condensation process. Protostellar separation of gas and grains will affect stellar metal content.

Until recently any comparison of solar and meteoritic abundances suffered from large uncertainties of the solar data, largely caused by erroneous laboratory measurements of atomic oscillator strengths. Refined experimental techniques helped to improve this situation. The present state is illustrated in Figure 1 , which is based upon 56 solar analyses. Error limits for these elements are typically $\pm 40 \%$ and should not exceed a factor of two. 1 The correlation between the sun and carbonaceous chondrites is close and comprises elements of very different character. Solar Li, Be, and B may have been affected by nuclear reactions with protons in the sun.

Few elements at present permit to achieve higher accuracy. $\mathrm{Na}, \mathrm{Mg}, \mathrm{S}$, and $\mathrm{Ca}$ appear favorable. Being abundant, they are wel1-represented in the solar spectrum. As neutrals or ions they possess simple electronic configurations, allowing quantum-mechanical calculation of oscillator strengths of superior quality. Spectrum analysis should employ the same solar model and appropriate line-broadening data for each element.

Results for $\mathrm{Na}$ and $\mathrm{Ca}$ are available (Holweger 1971,1972). The lines of Ca+ (excluding $\lambda 3968$ ) and the autoionizing 1 ines lead to a solar abundance. $10 \mathrm{~g}$. Ca $=$ 6.36 , on the scale where $\log \varepsilon_{H}=12$. The sodium abundance deduced from all lines not involving $\mathrm{D}$ terms is $\log \mathrm{E}_{\mathrm{Na}}=6.32$. New results are given here for sulfur. All unblended, sufficient ly weak solar S I lines were selected which involve only unperturbed atomic levels, as specified by Lambert and Warner (1968). This permitted to use their calculated oscillator strengths. Measurements (SchulzGulde 1971) provide two additional blue lines. The data summarized in Table I lead to a sulfur abundance, $\log E_{S}=7.19$. The small scatter among individual lines, in particular those having $W>5 \mathrm{~m} \AA$, supports the prediction by Lambert and Warner that the oscillator strengths are reliable. We note also close agreement

${ }^{1} A$ table of the compiled solar and meteoritic abundances, including references, is available from the author. An independent compilation has been published by Ross and Aller (1976). 
HOLWEGER

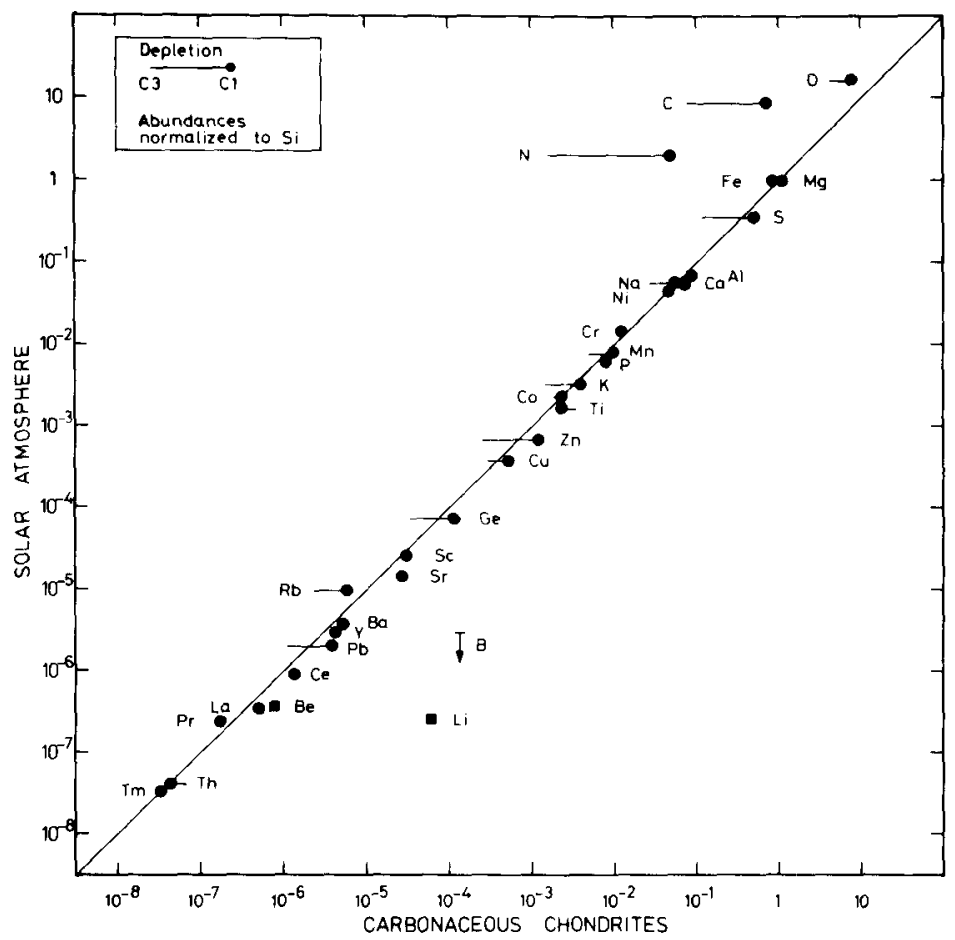

Figure 1. Comparison of elemental abundances in the sun with those in carbonaceous chondrites.

T'ABLE I

SOLAR SULFUR LINES USED FOR ABUNDANCE DETERMINATION*

\begin{tabular}{crrr|rrrr}
\hline$\lambda$ & $w$ & $\log g f$ & $\log \varepsilon$ & \multicolumn{1}{c}{$\lambda$} & W & $\log \mathrm{gf}$ & $\log \varepsilon$ \\
\hline 4694.13 & 11.8 & -1.80 & 7.32 & 8670.24 & 4.5 & -0.93 & 7.07 \\
4695.44 & 8.2 & -1.95 & 7.28 & 8670.68 & 11.0 & -0.58 & 7.16 \\
6052.66 & 10.9 & -0.63 & 7.24 & 8671.35 & 8.0 & -0.69 & 7.11 \\
6743.58 & 10.0 & -0.66 & 7.24 & 8693.14 & 1.5 & -1.42 & 7.08 \\
6743.79 & & & & 8693.99 & 12.5 & -0.56 & 7.21 \\
6756.98 & 17.9 & -0.29 & 7.19 & 8694.71 & 33.0 & +0.03 & 7.24 \\
6757.16 & 17 & & & 10456.76 & 64.0 & -0.44 & 7.22 \\
7686.09 & 3.8 & -1.07 & 7.19 & 15469.81 & 55.0 & -0.25 & 7.17 \\
\hline
\end{tabular}

*Wavelength $(\AA) ;$ line strength $W$ (milliangstroms) at center of solar disk (width of idealized box-shaped zero-intensity line absorbing the same energy): oscillator strength $\mathrm{gf}$; abundance $\varepsilon$ deduced from solar line (atoms $/ 10^{12} \mathrm{H}$ atoms). 
with their final result, $\log \mathrm{F}_{\mathrm{S}}=7.21$, showing that solar-model uncertainties play only a minor role. To eliminate these uncertainties as far as possible we replace hydrogen by a reference element having weak lines. Ca is to be preferred to $\mathrm{Si}$, regarding oscillator strengths.

Accordingly, the hest solar abundance data we can obtain at present probably are the ratios $\mathrm{Na} / \mathrm{Ca}=0.91$ and $\mathrm{S} / \mathrm{Ca}=6.8$. (A preliminary result for magnesium is $\mathrm{Mg} / \mathrm{Ca}=16,2)$. Residual uncertainties amount go about $\pm 15 \%$. Corresponding data for $\mathrm{Cl}$ chondrites are: $\mathrm{Na} / \mathrm{Ca}=0.80, \mathrm{~S} / \mathrm{Ca}=6.9$, and $\mathrm{Mg} / \mathrm{Ca}=14.7$ (Nichiporuk and Moore 1974; Moore 1971, see Mason 1971). A scatter of about $\pm 10 \%$ is inferred from individual analyses.

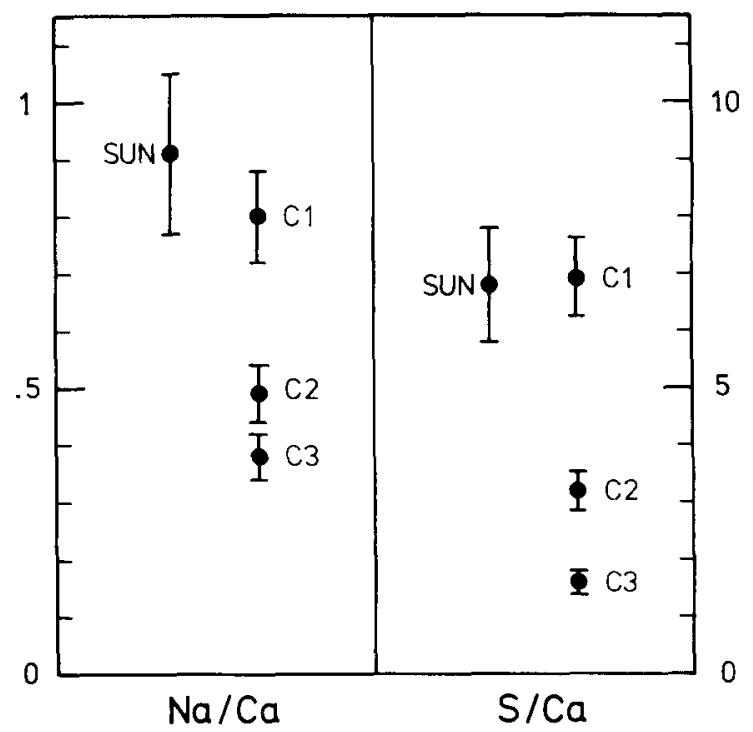

Figure 2. Comparison of the solar abundance ratios $\mathrm{Na} / \mathrm{Ca}$ and $\mathrm{S} / \mathrm{Ca}$ with those in carbonaceous chondrites.

These results (Figure 2) reveal a remarkably close relationship between the sun and carbonaceous chondrites. Clearly type l matches the solar composition best. Obviously C1 matter is a wel1-preserved condensate from parts of the solar nebula whose composition was very close to solar.

This close relationship would not be expected if $\mathrm{Cl}$ chondrites largely consisted of interstellar grains that escaped vaporization. The depletion pattern observed in the gas phase of interstellar clouds (e.g., Morton and Hu 1975; Knapp et al. 1976) suggests that the grains containing the missing elements will indeed somehow resemble $\mathrm{Cl}$ matter, but will be peculiar at least by a much lower $\mathrm{S} / \mathrm{Ca}$, because sulfur is left in the gas.

Will interstellar gas and grains be lumped together into stars without any separation? A moderate fractionation occurring prior to, or during star formation will affect the stellar metal/hydrogen ratios. This could explain why cluster stars, though originating from the same cloud, differ in metal content.

\section{REFERENCES}

Holweger, H. 1971, Astr. and Ap., 10, 128. 


\section{HOLWEGER}

Holweger, H. 1972, Sol. Phys. 25, 14 .

Knapp, G. R., Kuiper, T.B.H., and Brown, R. L. 1975, Astrophys. J., $206,109$. Lambert, D. L., and Warner, B. 1968, M.N.R.A.S., 138, 18J.

Mason, B. (ed.). 1971, Handbook of Elemental Abundances in Meteorites, (New York: Gordon and Breach).

Morton, D. C., and Hu, E. M. 1975, Astrophys. J., 202, 638.

Nichiporuk, W., and Moore, C. B. 1974, Geochim. Cosmochim. Acta, 38, 1691.

Ross, J. E., and Aller, L. H. 1976, Science, 191, 1223.

Schulz-Gulde, E. 1971, Zs. F. Phys., 245, 308. 